\title{
Photometric observation of the transiting exoplanet WASP-1b
}

\author{
Xiao-bin Wang ${ }^{1}$, Andrew Collier Cameron ${ }^{2}$, Sheng-hong $\mathrm{Gu}^{1}$ and \\ Li-yun Zhang ${ }^{1}$ \\ ${ }^{1}$ National Astronomical Observatories/Yunnan Observatory, CAS, \\ Kunming 650011, China \\ email: wangxb@ynao.ac.cn \\ ${ }^{2}$ School of Physics and Astronomy, University of St Andrews, \\ Fife KY16 9SS, UK \\ email: acc4@st-and.ac.uk
}

\begin{abstract}
The extra-solar planet WASP-1b, discovered by SuperWASP consortium in 2006, was observed through R filter using the 1-meter telescope with CCD camera at Yunnan Observatory on November 11, 2006. The systematic errors in photometric data were reduced by means of Tamuz et al. (2005) and Collier Cameron et al. (2006)'s algorithms. In order to estimate the parameters of the system, the MCMC (Markov Chain Monte Carlo) analysis is applied to fit the observed light curve. The following parameters of the system are derived: $R_{p}=1.44 \mathrm{R}_{J}$, $M_{p}=0.88 \mathrm{M}_{J}, R_{*}=1.52 \mathrm{R}_{\odot}, a=0.0396 \mathrm{AU}$. The new parameters of the planet imply its low density, which agrees to the previous results.
\end{abstract}

Keywords. exoplanet, transit, photometry

\section{Introduction}

Photometric observation of transiting exoplanet can provide us the size and mass of the host star and the planet. Furthermore, we can know the density of the planet, which is very important for understanding the formation of stellar system with planets. The SuperWASP project (Pollacco et al. 2006) is UK wide-field survey for exoplanet transits. The extra-solar planet WASP-1b was discovered by the WASP consortium (Collier Cameron et al. 2007a) in 2006. Later, the follow-up photometric and spectroscopic observations (Charbonneau et al. 2007, Shporer et al. 2007, Stempels et al. 2007) for the system were made, and the basic parameters of the system were derived.

\section{Observation}

We made the photometric observation for WASP-1b during its transit period using the 1-meter telescope at Yunnan Observatory on November 11, 2006. The observational data were gathered by using a $1024 \times 1024$ pixel $\mathrm{CCD}$ detector through the $\mathrm{R}$ filter. The scale of one pixel is $0.38^{\prime \prime}$, the field of view of the camera is about $6.5^{\prime} \times 6.5^{\prime}$. The observation for this transit event was done at air mass less than 1.2 with an exposure time of 30 seconds.

\section{Data reduction}

The observed CCD images are reduced with the IRAF package, including the bias subtraction, flat-fielding and cosmic ray removal. The instrumental magnitude of the 
WASP-1 system and 5 comparison stars in the field of view are measured by using APPHOT sub-package of IRAF through an aperture with radius of 6.5 ". Finally, the observed light curve contains 312 data points.

For the amplitude of light variation due to transit of a planet is small, the high-quality photometric data are necessary for searching new exoplanets and re-determining the parameters of known systems. Here, we have applied Tamuz et al. (2005) and Collier Cameron et al. (2006)'s algorithms to minimize the systematic effects in photometric data so as to enhance the signal to noise ratio of the light curve. Fig. 1 shows the light curve on Nov. 11, 2006 after removing the systematic effects.

\section{Photometric parameters}

Assuming the planet's orbit is circular, we model the flux of the transiting system with the parameters $\left\{T_{c}, p, \Delta F, t_{T}, b, M_{*}\right\}$ considering the 4-coefficient limb-darkening law of Claret (2000). The limb darkening coefficients for the R-band are interpolated from Claret (2000)'s table according to the effective temperature of the host star. The basic parameters of the host star are adopted from the recent results of Stempels et al. (2007). All observed data points are involved in MCMC (Markov Chain Monte Carlo) analysis to search the optimal parameters $\left\{T_{c}, p, \Delta F, t_{T}, b, M_{*}\right\}$ under the condition of minimizing the quantity $\chi^{2}$ (Collier Cameron et al. 2007b). Here, the chi-squared $\chi^{2}=\sum_{j}\left(m_{j}-\mu_{j}\right)^{2} / \sigma_{j}^{2}, m_{j}$ and $\mu_{j}$ are observed and model flux, respectively. For the span of our observational data is short, only five parameters $\left\{T_{c}, \Delta F, t_{T}, b, M_{*}\right\}$ are analyzed in practice.
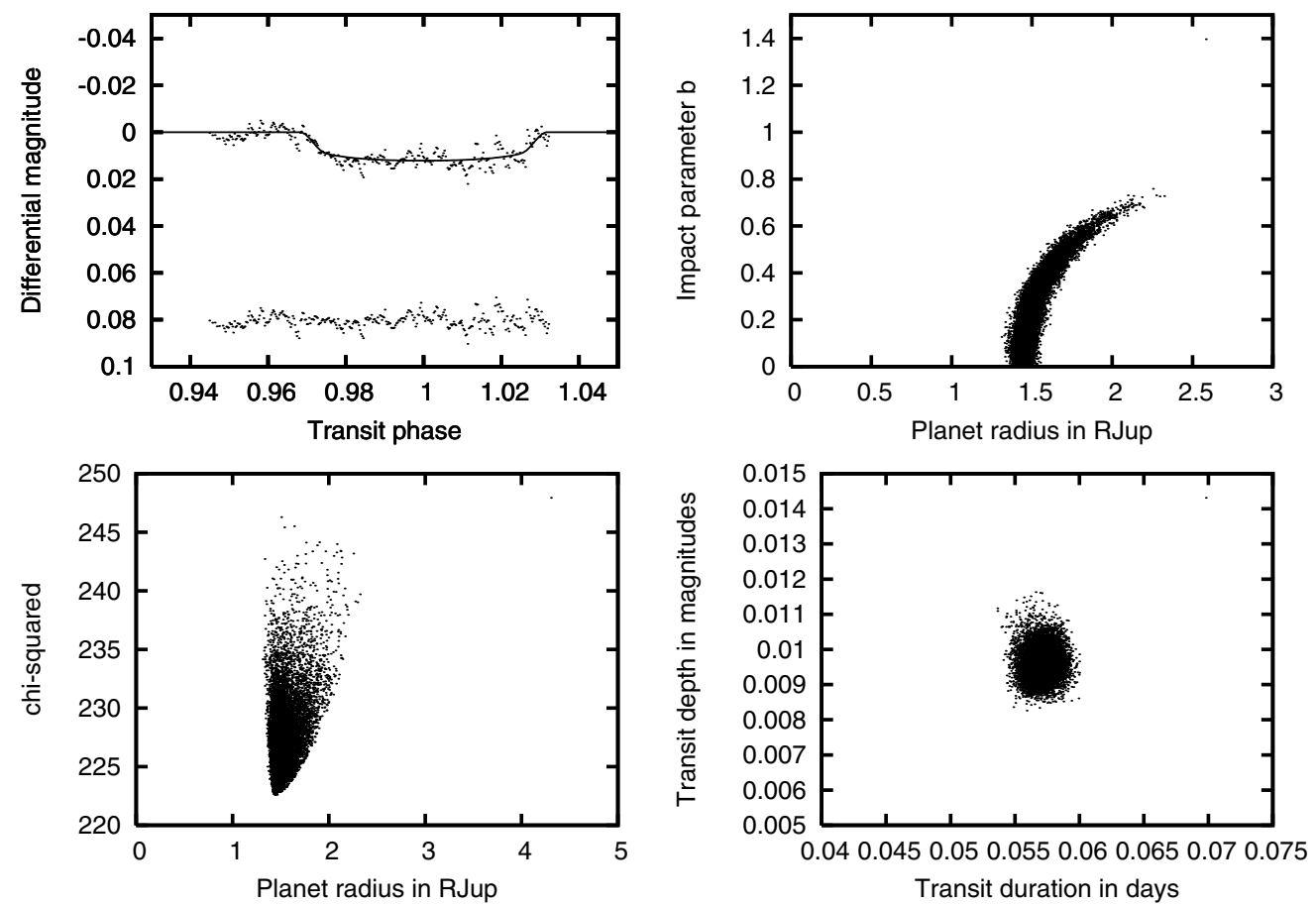

Figure 1. Upper-left panel: Light curve of WASP-1 system and fitting. Lower-left panel: The radius of planet (in unit of radius of Jupiter) vs chi-squared. Upper-right panel: The relation between radius of planet and impact parameter. Lower-right panel: The relation between transit duration and transit depth. 
Table 1. The optimal parameters derived using the MCMC method.

\begin{tabular}{ll}
\hline Transit epoch $T_{c}$ [HJD] & $2454051.1087^{+0.0013}-0.0006$ \\
Transit depth $\Delta F$ & $0.0094^{+0.0006}-0.0002 \mathrm{mag}$ \\
Transit width $t_{T}$ & $0.1436^{+0.0020}-0.0021$ days \\
Impact parameter $b$ & $0.012^{+0.452}-0.095 \mathrm{R}_{*}$ \\
Orbital separation $a$ & $0.0396^{+0.0003}-0.0003 \mathrm{AU}$ \\
Orbital inclination $i$ & $89.87^{+-0.98}-5.25$ degrees \\
Stellar radius $R_{*}$ & $1.52^{+0.19}--0.006 \mathrm{R}_{\odot}$ \\
Planet radius $R_{p}$ & $1.44^{+0.22}-0.002 \mathrm{R}_{J}$ \\
Stellar mass $M_{*}$ & $1.30^{+0.03}-0.03 \mathrm{M}_{\odot} \odot$ \\
Planet mass $M_{p}$ & $0.879^{+0.014}-0.013 \mathrm{M}_{J}$ \\
Orbital period $p$ & 2.51996 days \\
\hline
\end{tabular}

With the stellar mass of $1.30 \mathrm{M}_{\odot}$ (Stempels et al. 2007) and orbital period of 2.51996days (Shporer et al. 2007), the optimal values of other parameters are derived (see Fig. 1). Table 1 lists the optimal parameters of this system. From our new simulated parameters, WASP-1b is a low density planet among known transiting exoplanets, which is similar to the results derived by other authors (Charbonneau et al. 2007, Shporer et al. 2007).

\section{Acknowledgements}

This work is partly supported by NSFC under grant No. 10673027.

\section{References}

Charbonneau, D., Joshua, N., Mark, E. et al. 2007, ApJ, 658, 1322

Claret, A. 2000, A\&A, 363, 1081

Collier Cameron, A. et al. 2006, MNRAS, 373, 799

Collier Cameron, A., Bouchy, F., Hebrard, G. et al. 2007a, MNRAS, 375, 951

Collier Cameron, A., Wilson, D. M., West, R. G. et al. 2007b, MNRAS, 380, 1230

Pollacco, D. L. et al. 2006, PASP, 118, 1407

Shporer, A., Tamuz, O., Zucher, S. \& Mazeh, T. 2007, MNRAS, 376, 1296

Stempels, H. C., Collier Cameron, A., Hebb, L. et al. 2007, MNRAS, 379, 773

Tamuz, O., Mazeh, T. \& Zucker, S. 2005, MNRAS, 356, 1466 